\title{
Studies for the evaluation of the performance of the Time over Threshold technique for the digitization of the signal of a Very Large Volume Neutrino Telescope
}

\author{
G. Bourlis* , Leisos, A. G. Tsirigotis and S. E. Tzamarias \\ School of Science \& Technology, Hellenic Open University \\ Tsamadou 13-15, Patra, Greece \\ E-mail: bourlis@eap.gr, leisos@eap.gr, tsirigotis@eap.gr, \\ tzamaria@eap.gr
}

\begin{abstract}
KM3NeT ( $\mathrm{km}^{3}$ Neutrino Telescope) is planned to be a deep-sea multidisciplinary observatory in the Mediterranean Sea that will provide innovative science opportunities spanning Astroparticle Physics and Earth and Sea Science. KM3NeT will consist of several hundreds of vertical structures, which carry photo-sensors and devices for calibration and environmental measurements. The photo-sensor unit is a digital optical module (DOM) consisting of a 17-inch diameter, pressure resistant glass sphere, housing 31 3-inch photomultiplier (PMT) tubes. The front-end electronics is based on the use of the Time over Threshold (ToT) as the main signal processing technique. The track reconstruction techniques applied to the KM3NeT data, use the arrival times of the first photon on each PMT and the deposited charge. We report on the performance of the ToT technique in reconstructing the PMT signal characteristics, in particular the first photon arrival time and the charge deposited on the PMT. For this study, we have used simulated data generated with the HOURS (HOU Reconstruction \& Simulation) software package.
\end{abstract}

International Workshop on New Photon-detectors

June 13-15, 2012

LAL Orsay, France

${ }^{*}$ Speaker.

${ }^{\dagger}$ On behalf of the KM3NeT Consortium 


\section{Introduction}

KM3NeT ( $\mathrm{km}^{3}$ Neutrino Telescope) will be one of the world's largest particle detectors, built at the bottom of the Mediterranean Sea. It will search for neutrinos from distant astrophysical sources, such as gamma ray bursters, supernovae and colliding stars, and will contribute in the search for dark matter in the Universe. KM3NeT [1] is in its preparatory phase and is building on experience from 3 current Mediterranean projects: ANTARES, NEMO and NESTOR. The proposed deep-sea infrastructure will also house instrumentation from Earth and marine sciences: Oceanology, Marine Biology, Environmental Science, Geology and Geophysics.

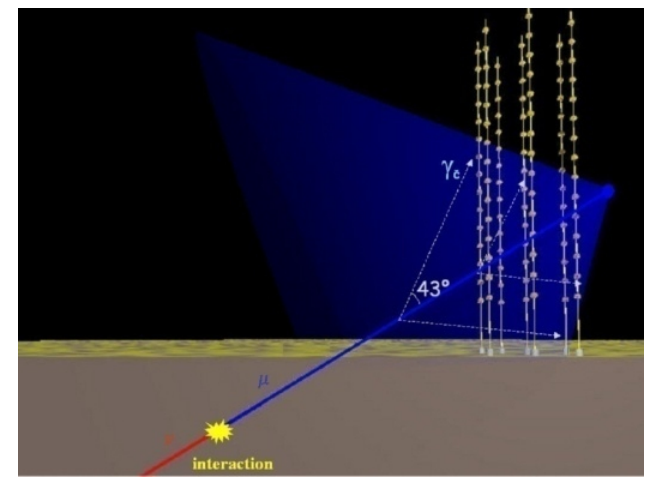

Figure 1: Principle of operation of an underwater neutrino telescope

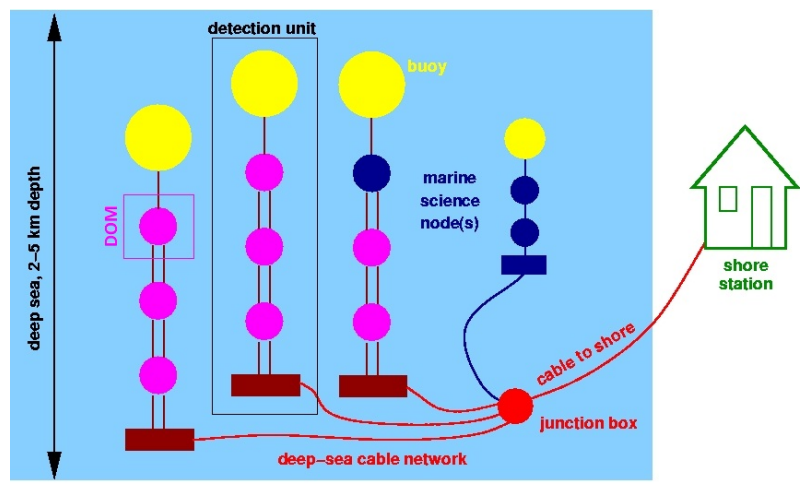

Figure 2: KM3NeT underwater neutrino telescope layout

The weak nature of neutrino interactions preserves their energy and directionality potentially allowing them to illuminate parts of the Universe opaque to charged particles and EM radiation. In order to observe the predicted fluxes, one must instrument $\mathrm{km}^{3} \mathrm{~s}$ of material sensitive to the resulting secondary radiation. The deep Mediterranean permits observation of Cherenkov photons (Figure 1) with attenuation lengths of up to $50 \mathrm{~m}$. KM3NeT is composed of a number of vertical structures (the Detection Units) (Figure 2), which carry photo-sensors and devices for calibration and environmental measurements [1]. The basic photo-sensor unit is a "Digital Optical Module (DOM)" housing several photomultiplier (PMT) tubes, their high-voltage bases and their interfaces to the nanosecond timing precision data acquisition system.

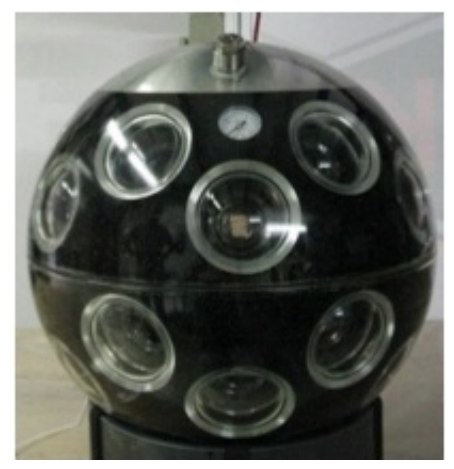

Figure 3: The KM3NeT Digital Optical Module

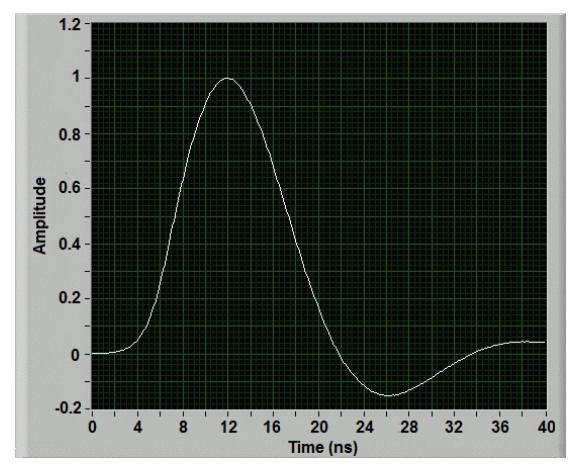

Figure 4: Typical PMT pulse (amplitude normalized to a peak of $1 \mathrm{~V}$ ) 
Each DOM consists of 31 3" PMTs ( $30 \%$ maximum QE) inside a 17" glass sphere with 31 bases (total consumption of $6.5 \mathrm{~W}$ ) and includes a cooling shield and stem (Figure 3). The photocathode area per DOM is $1260 \mathrm{~cm}^{2}$. The first full prototype is under extended testing in Nikhef. For the following analysis, pulses (Figure 4) from the candidate ET Enterprise Ltd. D783FL PMT are used [2].

The ToT technique is based on the time-tagging of the leading and trailing edge of the PMT signal above a number of thresholds. For the KM3NeT one threshold will be utilized. The ToT functionality is embedded in the PMT bases, where the PMT signals are converted to a time over threshold signal, with the use of an adjustable threshold comparator [3]. These signals are driven to the DOM central logic board. The ToT technique offers the advantage of significantly reduced amount of data to be transferred to shore, while it does not require complex electronics for its implementation.

\section{Muon track reconstruction and energy estimation}

The muon track is reconstructed from the arrival times of the Cherenkov photons on the PMTs and the positions of the PMTs. The energy is estimated from the deposited charge on each DOM. The charge probability distribution function, $\mathrm{P}$, (that depends on the muon energy $\mathrm{E}$, the distance from the track D and the PMT orientation with respect to the wavefront of the emitted Cherenkov light $\theta$ ) is estimated via simulation (Figure 5). The following Likelihood function is formed

$$
L(E)=\ln \left(\prod_{i=1}^{N_{h i t}} P\left(Q_{i, \text { data }} ; E, D, \theta\right) \prod_{i=1}^{N_{\text {nohit }}} P(0 ; E, D, \theta)\right)
$$

and maximized with respect to the muon energy.

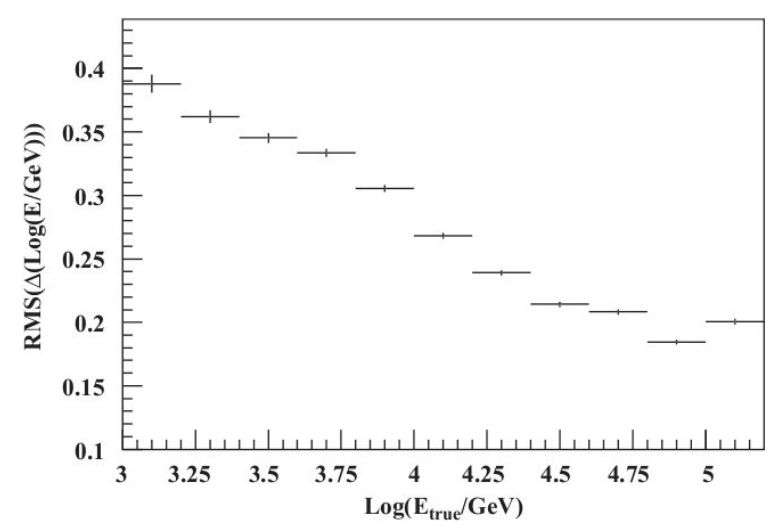

Figure 5: Energy resolution as a function of the energy, considering the PMT charge known with zero error.

\section{Slewing correction estimation}

For the timing of the signals, using the ToT technique, the time of the crossing of the lower threshold is utilized. This results in a bias of the timing of the pulses (slewing), which depends 
on the pulse amplitude. The timing of the pulses is corrected for the slewing effect, by developing parametrizations of the slewing (Figure 6) and the error of the slewing estimation as a function of the time over threshold (tot) values. Simulated pulses have been generated for muons of energy $1 \mathrm{TeV}-100 \mathrm{TeV}$, with an $E^{-2}$ spectrum at isotropical directions and uniform distances from the DOM, using the HOURS (Hellenic Open University Reconstruction \& Simulation) software package [4]. The results of the analysis, using one threshold of $0.3 \mathrm{pe}$, show that the slewing effect can be estimated with a resolution of $5.5 \%$ (Figure 7).
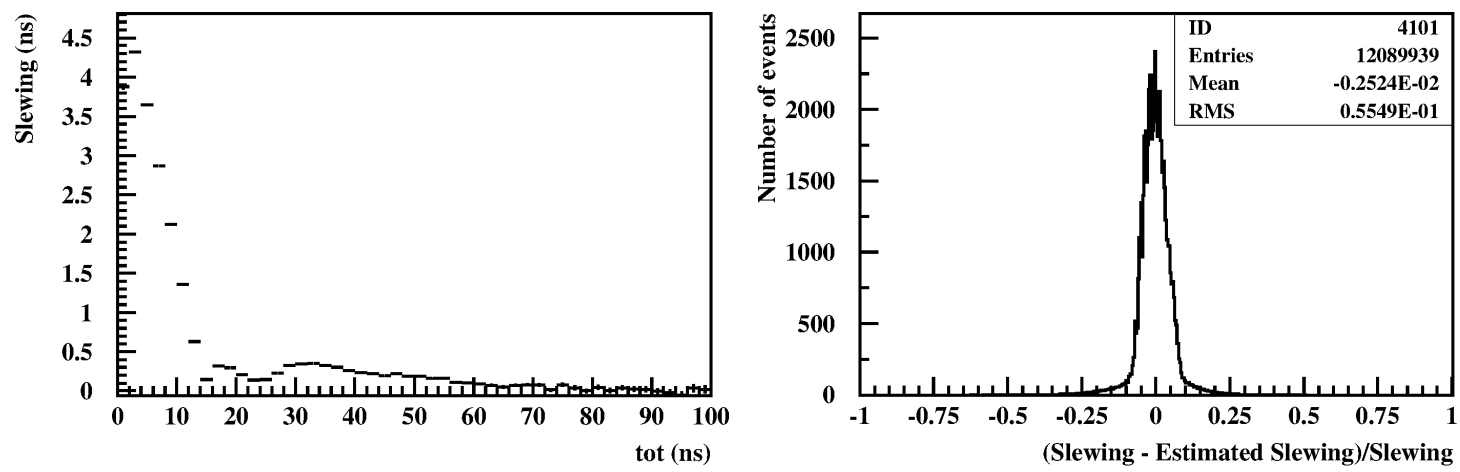

Figure 6: Slewing parametrization as a function of Figure 7: Percentage error of the slewing estimathe values of tot. tion.

The use of the slewing parametrizations does not increase the statistical error in estimating the arrival time of the pulses. This is evident if the expected arrival times of the photons (that is calculated geometrically) is compared, with the true (simulated) arrival times of the photons, the arrival times estimated using the threshold crossing and the arrival times estimated using the threshold crossing corrected for the slewing effect (Figure 8).

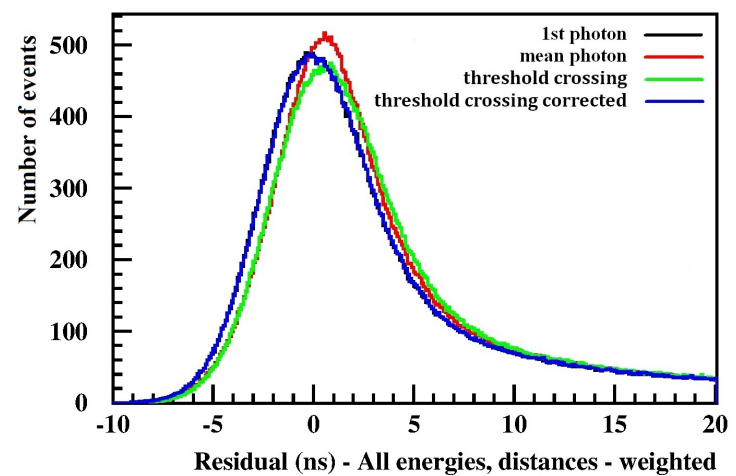

Figure 8: Difference between the expected arrival times of the first photon and a) the true (simulated) arrival times of the first photon (black line which coincides with the blue one), b) the mean arrival times of the photons (red), c) the arrival times estimated using the threshold crossing (green) and d) the arrival times estimated using the threshold crossing corrected for the slewing effect (blue).

\section{PMT and DOM charge estimation}

The use of the ToT technique with only one threshold, does not allow the parametrization of the charge of each PMT with a good resolution (around 60\% for pulses more than 2pe) (Figure 9). 
This is due to the non standard shape of the PMT pulses (Figure 10). If multiple thresholds are used the resolution improves significantly, e.g. for six thresholds it drops to around 8\% [5]. The peak at around $25 \mathrm{~ns}$ corresponds to large number of photons arriving almost synchronously at the PMT. For these pulses the tot values do not vary more than $5 \mathrm{~ns}$, while the charge can reach more than 50pe.

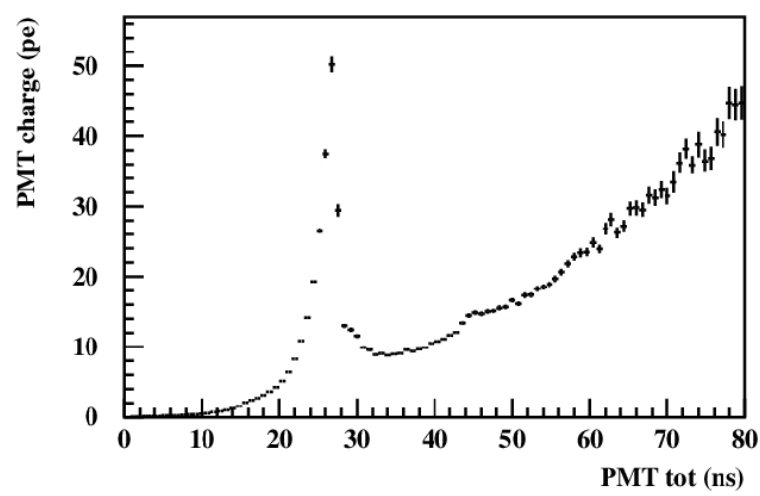

Figure 9: PMT charge parametrization.

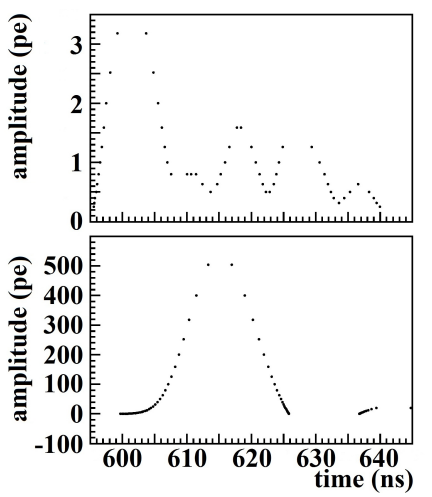

Figure 10: Different PMT pulse shapes.

However, if the DOM total charge is parametrized as a function of the sum of the tot values of the PMTs (Figure 11), the DOM total charge can be estimated with a resolution of 20\% (Figure 12). The performance of the energy reconstruction technique, as described in Section 2, in general depends on the DOM charge resolution. However, employing the 20\% DOM charge resolution in the energy reconstruction technique, the change in the energy resolution lies within the statistical errors.

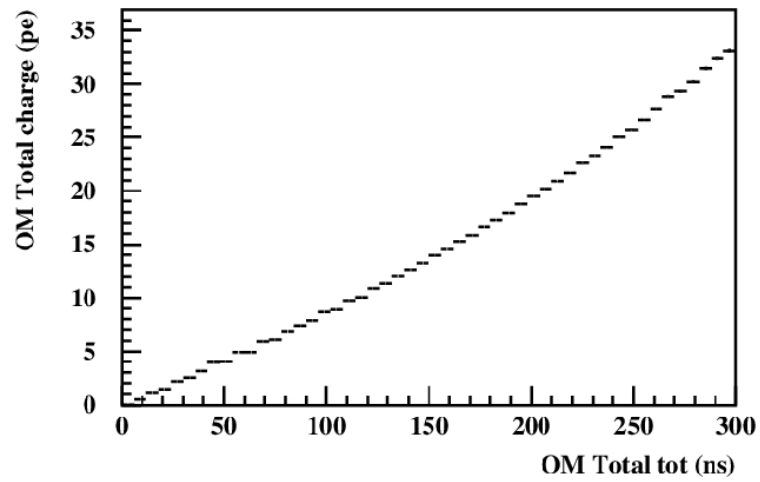

Figure 11: DOM total charge parametrization as a function of the sum of the tot values of the PMTs.

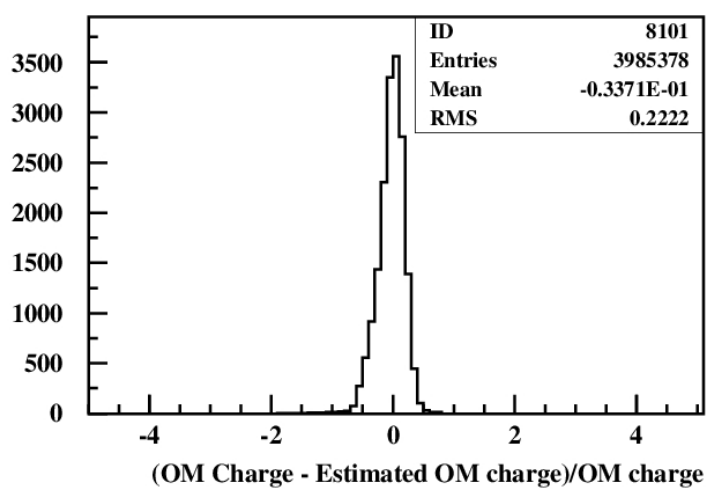

Figure 12: Percentage error of the DOM total charge estimation.

The DOM charge estimation can be improved further, if the number of hit PMTs and the correlations between the arrival times of the pulses at each hit PMT of the DOM are taken into account. Thus, the pulses of different shape are categorized according to the number of hit PMTs and the RMS of the arrival times of the pulses of the PMTs. Then, different parametrizations of the DOM total charge are used and the results are presented in Figure 13. 


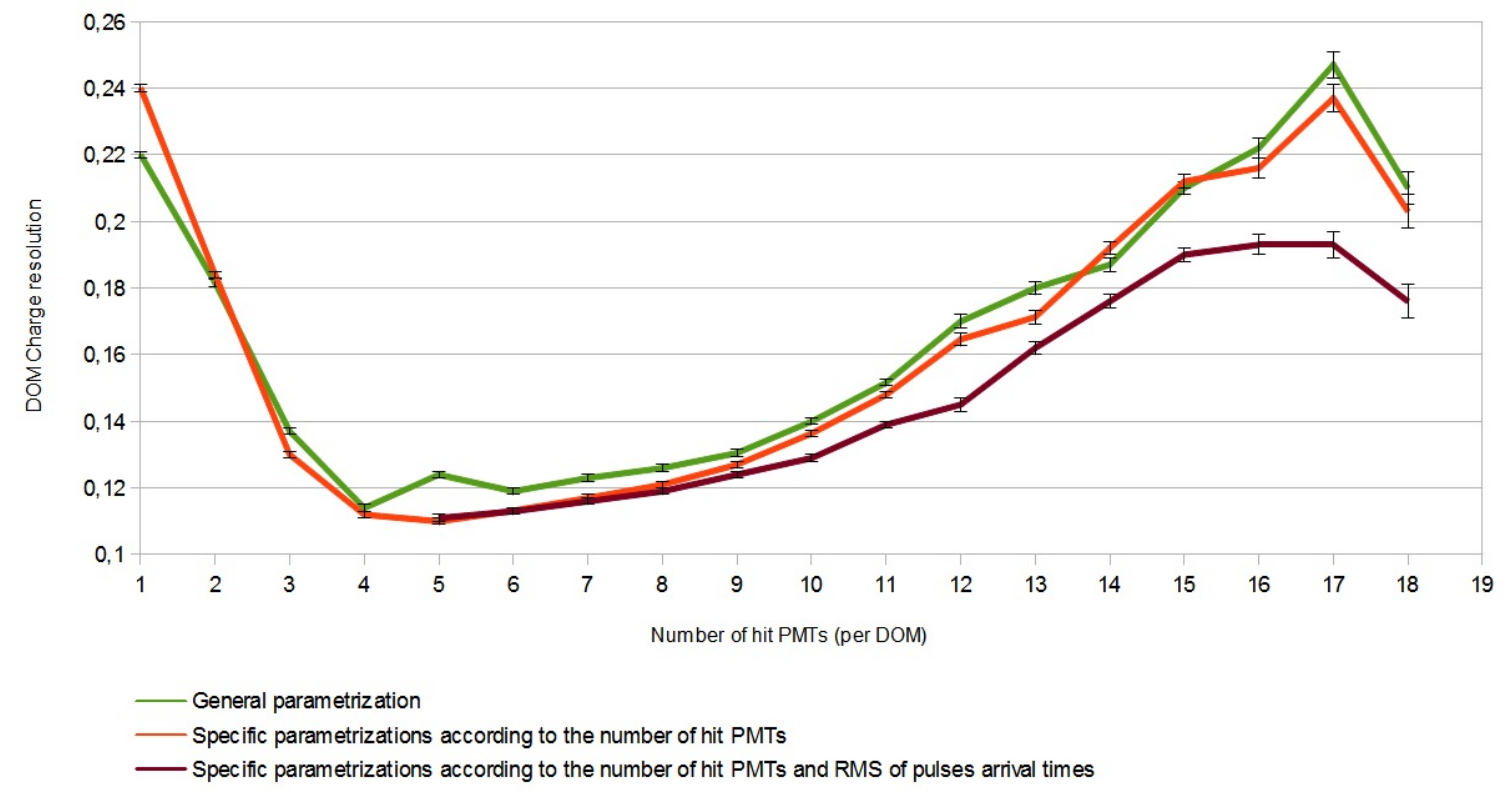

Figure 13: DOM charge resolution as a function of the number of hit PMTs of the DOM, when: a general DOM charge parametrization is used (green), specific DOM charge parametrizations for different numbers of hit PMTs are used (red) and specific DOM charge parametrizations for different numbers of hit PMTs and areas of the RMS of the pulse arrival times are used (brown).

\section{Conclusions}

The use of the ToT technique as the main signal processing technique for the KM3NeT DOMs, does not add significantly to the statistical error of the timing of the pulses, while the slewing effect can be estimated with a resolution of around 5\%. The DOM charge can be estimated using the ToT technique with only one threshold with a resolution of $20 \%$, which is adequate for the energy reconstruction. Studies are ongoing for the improvement of the DOM charge resolution, based on the correlations of the arrival times of the PMTs of each DOM.

\section{References}

[1] http://www.km3net.org; Technical Design Report for a Deep-Sea Research Infrastructure in the Mediterranean Sea Incorporating a Very Large Volume Neutrino Telescope (http://km3net.org/TDR/TDRKM3NeT.pdf)

[2] Q. Dorosti et al., Performance of Photo-Sensors for KM3NeT, to appear in the VLVnT11 proceedings.

[3] KM3NeT consortium, Technical description of the PPM of the KM3NeT detection unit (http://km3net.org/PPM/KM3NeT_FL_REP_PPM-description.pdf)

[4] A. G. Tsirigotis, et al., HOU Reconstruction \& Simulation (HOURS): A complete simulation and reconstruction package for Very Large Volume underwater neutrino Telescopes, Nucl. Instr. and Meth. A. 626-627 (2011) S185-S187, (http://dx.doi.org/10.1016/j.nima.2010.06.258)

[5] G. Bourlis, A.Leisos, A. G. Tsirigotis, S.E.Tzamarias, Use of the Multi-Time over Threshold electronics to digitize signals from VLVnT, Nucl. Instr. and Meth. A. 626-627 (2011) S163-S165, (http://dx.doi.org/10.1016/j.nima.2010.04.153) 